\title{
A dividing method utilizing the best multiplication in affine arithmetic
}

\author{
Shinya Miyajima ${ }^{\text {a) }}$ and Masahide Kashiwagi \\ School of Science and Engineering, Waseda University, 61-404, 3-4-1 Okubo, \\ Shinjuku-ku, Tokyo, 169-8555 Japan \\ a) shinya_miyajima@fuji.waseda.jp
}

Abstract: Affine arithmetic (AA) is a variant of interval arithmetic. In $\mathrm{AA}$, it is difficult to realize the efficient nonlinear binomial operations. The purpose of this paper is to propose a new dividing method which consists of a reciprocal and the best multiplication. And the performances of the new method and the conventional method are compared through some numerical examples.

Keywords: interval arithmetic, affine arithmetic, division, the best multiplication

Classification: Science and engineering for electronics

\section{References}

[1] Shin'ichi Oishi, "Numerical Calculation," SHOKABO, Tokyo, 1999 (in Japanese).

[2] Shin'ichi Oishi, "Verified Numerical Computation," CORONA PUBLISHING, Tokyo, 2000 (in Japanese).

[3] Alefeld, G. and Herzberger, J., "Introduction to Interval Computations," Academic Press, New York, 1983.

[4] A. Neumaier, "Interval methods for systems of equations," Cambrige University Press, 1990.

[5] Kiyotaka Yamamura, Ai Tokue, and Hitomi Kawata, "Finding All Solutions of Nonlinear Resistive Circuits by Interval Analysis," IEICE Trans. Fundamentals, vol. J79-A, no. 10, pp. 1692-1699, Oct. 1996 (in Japanese).

[6] Kiyotaka Yamamura and Shigeru Tanaka, "Improvement of the contraction-type LP test algorithm for finding all solutions of piecewiselinear resistive circuits," Int. J. Circuit Theory and Applications, vol. 29, no. 4, pp. 403-411, July 2001.

[7] Marcus Vinícius A. Andrade, João L. D. Comba, and Jorge Stolfi, "Affine Arithmetic," INTERVAL'94, St. petersburg (Russia), March 5-10, 1994.

[8] Luiz H. de Figueiredo and Jorge Stolfi, "Self-Validated Numerical Methods and Applications," Brazilian Mathematics Colloquium monograph, IMPA, Rio de Janeiro, Brazil; July 1997.

[9] Shinya Miyajima, Takatomi Miyata, and Masahide Kashiwagi, "A New Dividing Method in Affine Arithmetic," IEICE Trans. Fundamentals, vol. E86-A, no. 9, pp. 2192-2196, Sept. 2003.

[10] Shinya Miyajima, Takatomi Miyata, and Masahide Kashiwagi, "On the Best Multiplication of the Affine Arithmetic," IEICE Trans. Fundamentals, vol. J86-A, no. 2, pp. 150-159, Feb. 2003 (in Japanese). 


\section{Introduction}

Numerical computation with guaranteed accuracy (e.g. [1, 2]) means numerical methods which calculate not only numerical results but also mathematically rigorous error evaluation of the results. Most of those methods are realized by interval arithmetic (IA) (e.g. [3, 4]). At present, IA is applied in circuit analysis (e.g. $[5,6])$. Moreover, we are able to expect that IA contributes to the development of the other fields in engineering for electronics.

On the other hand, IA has an essential weak point that it causes unexpected overestimation of calculation results. The main reason of the phenomenon is that correlation between quantities are ignored.

In order to overcome this problem, affine arithmetic (AA) [7, 8], an extension of standard IA, was proposed in 1994. Although division in AA is improved in [9], a method which supplies better results than [9] exists in theory (but the method is not known). On the other hand, in multiplication in AA, the best multiplication which always supplies the best result was proposed in [10].

The purpose of this paper is to propose a new dividing method which consists of a reciprocal and the best multiplication. And the performances of the new method and the conventional method [9] are compared through some numerical examples.

\section{Affine Arithmetic}

AA $[7,8]$ is a variant of IA (e.g. $[3,4]$ ). AA keeps correlation between quantities. So AA is able to overcome the extreme increase of interval width often observed in standard IA. In AA, a quantity $x$ is represented as the following affine form:

$$
x=x_{0}+x_{1} \varepsilon_{1}+\cdots+x_{n} \varepsilon_{n} .
$$

Here, coefficients $x_{i}(i=0,1, \cdots, n)$ are known real numbers, and $\varepsilon_{i}(i=$ $1, \cdots, n)$ are symbolic real variables whose values are unknown but assumed to lie in the interval $[-1,1]$. Only coefficients $x_{i}(i=0,1, \cdots, n)$ are memorized in the computer. Remark that $n$, the number of the noise symbol $\varepsilon_{k}$, changes through the calculation.

In the case that a $m$ variables function $f\left(x_{1}, \cdots, x_{m}\right)$ is evaluated in AA, first given $m$ variables are initialized in the following affine forms by introducing $m \varepsilon$ s because they do not correlate each other.

$$
x_{1}=\frac{\bar{x}_{1}+\underline{x}_{1}}{2}+\frac{\bar{x}_{1}-\underline{x}_{1}}{2} \varepsilon_{1}, \cdots, x_{m}=\frac{\bar{x}_{m}+\underline{x}_{m}}{2}+\frac{\bar{x}_{m}-\underline{x}_{m}}{2} \varepsilon_{m}
$$

Note that $\left[\underline{x}_{k}, \bar{x}_{k}\right]$ is the domain of variables $x_{k}$. An affine form (1) is always able to be returned to a standard interval by the following formula:

$$
\left[x_{0}-\Delta, x_{0}+\Delta\right], \quad \Delta=\sum_{i=1}^{n}\left|x_{i}\right|
$$

For affine forms

$$
\begin{aligned}
& x=x_{0}+x_{1} \varepsilon_{1}+\cdots+x_{n} \varepsilon_{n} \\
& y=y_{0}+y_{1} \varepsilon_{1}+\cdots+y_{n} \varepsilon_{n}
\end{aligned}
$$


and a real number $\alpha$, rules of linear operations for AA is defined as $x \pm y=$ $\left(x_{0} \pm y_{0}\right)+\sum_{i=1}^{n}\left(x_{i} \pm y_{i}\right) \varepsilon_{i}, \quad x \pm \alpha=\left(x_{0} \pm \alpha\right)+\sum_{i=1}^{n} x_{i} \varepsilon_{i}, \quad \alpha x=\left(\alpha x_{0}\right)+\sum_{i=1}^{n}\left(\alpha x_{i}\right) \varepsilon_{i}$.

In a nonlinear unary operation $f(x)$ for affine form (1), we consider linear approximation of $f(x)$ and representation of the approximation error by introducing a new noise symbol $\varepsilon_{n+1}$. First, $X$, range of $x$ (domain of $f$ ) is calculated by the formula (3). Next, $a x+b$, linear approximation of $f(x)$ in $X$ is calculated (see the Fig. 1). And maximum approximation error $\delta$ is calculated as $\delta=\max _{x \in X}|f(x)-(a x+b)|$. By introducing a new noise symbol $\varepsilon_{n+1}$, the result of nonlinear unary operations is represented as follows:

$$
f(x)=a x+b+\delta \varepsilon_{n+1}=a\left(x_{0}+x_{1} \varepsilon_{1}+\cdots+x_{n} \varepsilon_{n}\right)+b+\delta \varepsilon_{n+1} .
$$

In nonlinear binomial operation $f(x, y)$ for affine forms $(4)$ and $(5)$, the same method as nonlinear unary operation is possible. First, $X$ and $Y$, range of $x$ and $y$ is calculated by the formula (3). Next, $a x+b y+c$, linear approximation of $f(x, y)$ in the rectangular domain $X \times Y$ is calculated. And maximum approximation error $\delta$ is calculated as $\delta=\max _{x \in X \times Y} \mid f(x, y)-(a x+$ $b y+c) \mid$. By introducing a new noise symbol $\varepsilon_{n+1}$, the result of nonlinear binomial operations is represented as follows:

$$
\begin{aligned}
f(x, y) & =a x+b y+c+\delta \varepsilon_{n+1} \\
& =a\left(x_{0}+x_{1} \varepsilon_{1}+\cdots+x_{n} \varepsilon_{n}\right)+b\left(y_{0}+y_{1} \varepsilon_{1}+\cdots+y_{n} \varepsilon_{n}\right)+c+\delta \varepsilon_{n+1}
\end{aligned}
$$

We define the best nonlinear operations as the nonlinear operations which minimize $\delta$, coefficient of a new noise symbol $\varepsilon_{n+1}$. In nonlinear unary operations, formula (6) is the best result if $a x+b$ is the best approximation of $f$ on $X$. However, in nonlinear binomial operation, formula (7) is not always the best result even if $a x+b y+c$ is the best approximation of $f$ on $X \times Y$. The reason of this matter is as follows: In the case that correlation between quantities exists (common $\varepsilon$ is included in both $x$ and $y$ ), point $(x, y)$ does not lie in all of the rectangular range $X \times Y$. Generally, the range that point $(x, y)$ lies, which is called "joint range," forms a convex polygon, which possesses $2 n$ sides and is symmetrical with respect to the point $\left(x_{0}, y_{0}\right)$. Therefore, in order to realize the best nonlinear binomial operations, $a x+b y+c$, the best linear approximation of $f(x, y)$ in the "joint range" have to be calculated. But to realize the best nonlinear binomial operations is difficult in general.

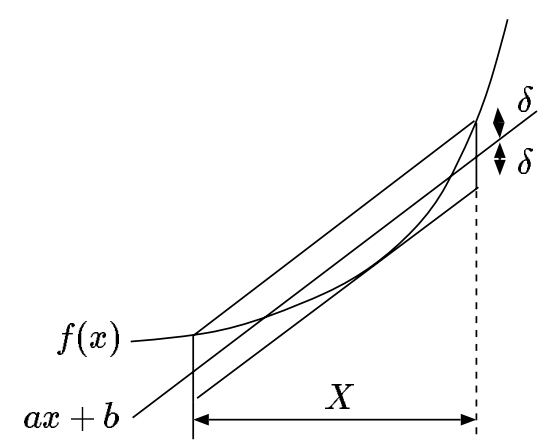

Fig. 1. Linear approximation of nonlinear function 


\section{New Dividing Method}

In this section, we propose the new dividing method. New dividing method consists of the combination of a reciprocal (nonlinear unary operation) and the best multiplication [10]. Concrete step of the new dividing method when we operate $x / y$ for affine forms $x$ and $y$ is as follows:

step1 Let $[y, \bar{y}]$ be the range of the affine form (5) calculated by the formula (3). According to the rule of the nonlinear unary operation, the reciprocal of the affine form (5) is calculated as follows:

$$
\begin{aligned}
\frac{1}{y}= & -\frac{1}{\frac{y}{y}} y_{0}+\frac{\underline{y}+\bar{y}+2 \sqrt{\underline{y} \bar{y}}}{2 \underline{y} \bar{y}}+\left(-\frac{y_{1}}{\underline{y} \bar{y}}\right) \varepsilon_{1}+\cdots+\left(-\frac{y_{n}}{\underline{y} \bar{y}}\right) \varepsilon_{n} \\
& +\frac{\underline{y}+\bar{y}-2 \sqrt{\underline{y} \bar{y}} \varepsilon_{n+1} \quad(0<\underline{y})}{2 \underline{y} \bar{y}} \\
\frac{1}{y}= & -\frac{1}{\frac{y}{y} y} y_{0}+\frac{\underline{y}+\bar{y}-2 \sqrt{\underline{y} \bar{y}}}{2 \underline{y} \bar{y}}+\left(-\frac{y_{1}}{y \bar{y}}\right) \varepsilon_{1}+\cdots+\left(-\frac{y_{n}}{\underline{y} \bar{y}}\right) \varepsilon_{n} \\
& +\frac{-\underline{y}-\bar{y}-2 \sqrt{\underline{y} \bar{y}}}{2 \underline{y} \bar{y}} \varepsilon_{n+1} \quad(\bar{y}<0) .
\end{aligned}
$$

To simplify the description, we put this reciprocal as follows:

$$
z=z_{0}+z_{1} \varepsilon_{1}+\cdots+z_{n} \varepsilon_{n}+z_{n+1} \varepsilon_{n+1} .
$$

step2 Let the domain $D$ be the joint range of $x$ and $z$, and let $d(x, z)$ be as follows:

$$
d(x, z)=x z-\left(z_{0} x+x_{0} z\right) .
$$

And calculate the following $d_{\max }$ and $d_{\min }$.

$$
\begin{aligned}
& d_{\max }=\max _{(x, z) \in D} d(x, z) \\
& d_{\min }=\min _{(x, z) \in D} d(x, z)
\end{aligned}
$$

The concrete way of calculating them are described in [10].

step3 Calculate $c$ and $\delta_{\mathrm{BM}}$ as follows:

$$
\begin{aligned}
c & =\frac{d_{\max }+d_{\min }}{2} \\
\delta_{\mathrm{BM}} & =\frac{d_{\max }-d_{\min }}{2} .
\end{aligned}
$$

At last, the result of the new dividing method is represented as follows:

$$
\frac{x}{y}=x \times z=z_{0} x+x_{0} z+c+\delta_{\mathrm{BM}} \varepsilon_{n+2} .
$$

step4 Terminate.

In the case that a correlation between variables is strong, we are able to expect that this method supply smaller $\delta$ than the conventional dividing method because the best multiplication exercise the efficiency even if two $\varepsilon \mathrm{s}$ $\left(x_{0} z_{n+1} \varepsilon_{n+1}\right.$ and $\left.\delta_{B M} \varepsilon_{n+2}\right)$ are added in one implementation. The calculating cost of this method is in proportion to $n^{2}$ where $n$ is the number of $\varepsilon$. 


\section{Numerical Examples}

In this section, performances of the new dividing method and the conventional dividing method [9] are compared through some numerical examples. Our computer environment is CPU: PentiumIV 3.06 GHz, memory: $1.00 \mathrm{~GB}$, OS: Microsoft Windows XP Professional and compiler: Microsoft Visual $\mathrm{C}++$ ver. 6.0.

Generally, because coefficient of added $\varepsilon$ i.e. $\delta$ does not be canceled through the calculation, overestimation of $\delta$ is considered the main cause which declines an performance of the interval evaluation. Therefore, the dividing method which calculate smaller $\delta$ progress the performance of the interval evaluation.

Hereafter, we explain about the numerical example concerning with the Table I and the Table II. Let $\delta_{\text {conv }}$ and $\delta_{\text {new }}$ be the $\delta$ s when we operated division $x / y$ for affine forms $x$ and $y$ by applying the method proposed in [9] and the new method. And let $t_{\text {conv }}$ and $t_{\text {new }}$ be the calculating costs $(\mu s)$ when we operated division $x / y$ for affine forms $x$ and $y$ by operating the method proposed in [9] and the new method. Next, we describe about the way of determining the affine forms $x$ and $y$. Let $x_{0}$ and $y_{0}$, central value of affine forms $x$ and $y$, be the random number which satisfies $-10 \leq x_{0} \leq$ 10, $2 \leq y_{0} \leq 10$. And let $x_{i}$ and $y_{i}$, coefficients of $\varepsilon_{i} \quad(1 \leq i)$, be the random numbers which satisfy the condition described in the Table I and the Table II. And let the ratios $R$ and $S$ be as follows:

$$
\begin{aligned}
R & =\frac{\delta_{\text {new }}}{\delta_{\text {conv }}} \\
S & =\frac{t_{\text {new }}}{t_{\text {conv }}} .
\end{aligned}
$$

The mean values of $R$ when we calculated them 10000 times are shown in the Table I. Similarly, the mean values of $S$ when we calculated them 10000

Table I. Mean values of $R$ when we calculate it 10000 times

\begin{tabular}{|c|c|c|}
\hline $\begin{array}{c}\text { condition of } \\
\text { coefficient of } \varepsilon_{i}\end{array}$ & $\begin{array}{c}\text { number } \\
\text { of } \varepsilon\end{array}$ & $\begin{array}{c}\text { mean value } \\
\text { of } R\end{array}$ \\
\hline \hline $\begin{array}{c}-0.1 \leq x_{i} \leq 0.1 \\
-0.1 \leq y_{i} \leq 0.1\end{array}$ & 4 & 1.048721 \\
\hline $\begin{array}{c}-0.1 \leq x_{i} \leq 0.1 \\
-0.1 \leq y_{i} \leq 0.1\end{array}$ & 11 & 1.042036 \\
\hline $\begin{array}{c}-0.1 \leq x_{i} \leq 0.1 \\
-0.1 \leq y_{i} \leq 0.1\end{array}$ & 4 & 1.217092 \\
\hline $\begin{array}{l}-0.01 \leq x_{i} \leq 0.01 \\
-0.01 \leq y_{i} \leq 0.01\end{array}$ & 11 & 0.778779 \\
\hline $\begin{array}{c}-0.001 \leq x_{i} \leq 0.001 \\
-0.001 \leq y_{i} \leq 0.001\end{array}$ & 4 & 1.175617 \\
\hline $\begin{array}{l}-0.001 \leq x_{i} \leq 0.001 \\
-0.001 \leq y_{i} \leq 0.001\end{array}$ & 11 & 1.209191 \\
\hline & \multicolumn{3}{|l}{}
\end{tabular}


Table II. Mean values of $S$ when we calculate it 10000 times

\begin{tabular}{|c|c|c|}
\hline $\begin{array}{c}\text { condition of } \\
\text { coefficient of } \varepsilon_{i}\end{array}$ & $\begin{array}{c}\text { number } \\
\text { of } \varepsilon\end{array}$ & $\begin{array}{c}\text { mean value } \\
\text { of } S\end{array}$ \\
\hline \hline $\begin{array}{c}-0.1 \leq x_{i} \leq 0.1 \\
-0.1 \leq y_{i} \leq 0.1\end{array}$ & 4 & 0.180875 \\
\hline $\begin{array}{c}-0.1 \leq x_{i} \leq 0.1 \\
-0.1 \leq y_{i} \leq 0.1\end{array}$ & 50 & 0.399976 \\
\hline $\begin{array}{l}-0.01 \leq x_{i} \leq 0.01 \\
-0.01 \leq y_{i} \leq 0.01\end{array}$ & 4 & 0.362751 \\
\hline $\begin{array}{l}-0.01 \leq x_{i} \leq 0.01 \\
-0.01 \leq y_{i} \leq 0.01\end{array}$ & 50 & 0.437102 \\
\hline $\begin{array}{l}-0.001 \leq x_{i} \leq 0.001 \\
-0.001 \leq y_{i} \leq 0.001\end{array}$ & 4 & 0.264369 \\
\hline $\begin{array}{c}-0.001 \leq x_{i} \leq 0.001 \\
-0.001 \leq y_{i} \leq 0.001\end{array}$ & 50 & 0.295267 \\
\hline
\end{tabular}

times are shown in the Table II.

Considering the general tendency of the size of $\delta$ which the each method supplies, by the Table I, we are able to confirm that $\delta_{\text {new }}$ is approximately equal to $\delta_{\text {conv }}$ although in the most examples in the Table I, $\delta_{\text {new }}$ is a little larger than $\delta_{\text {conv }}$. Therefore we are able to confirm that the new method supplies the interval evaluation approximately equal to the conventional method. And considering the general tendency of the each method's speed, by the Table II, we are able to confirm that $t_{\text {new }}$ is much smaller than $t_{\text {conv }}$. Therefore, we are able to confirm that the new method is much faster than the conventional method.

\section{Conclusion}

In this paper, we proposed a new dividing method in AA. The new method consists of a reciprocal and the best multiplication. And the performances of the new method and the conventional method were compared through some numerical examples. As the general tendency, the new method supplies the interval evaluation approximately equal to the conventional method. Moreover, the new method is much faster than the conventional method. We are able to expect that this result contributes to the progress of the IA's performance, which in turn contributes to the development of circuit analysis and the other fields in engineering for electronics.

\section{Acknowledgments}

This work is supported by Waseda University grant for special research projects (individual research 2003A-909). 